\title{
Implementation of strategies to improve programme effectiveness lead to an improvement in maternal and child health outcomes in South Africa
} \author{
M van der Merwe, ${ }^{5} \mathrm{RD}(\mathrm{SA}), \mathrm{MSc}$ (Nutr); P Barron, ${ }^{6} \mathrm{BCom}, \mathrm{FFCH}(\mathrm{SA})$ \\ ${ }^{1}$ United Nations Children's Fund (UNICEF), Pretoria, South Africa \\ ${ }^{2}$ South African Medical Research Council and University of Pretoria, Pretoria, South Africa \\ ${ }^{3}$ National Department of Health, Pretoria, South Africa \\ ${ }^{4}$ Department of Paediatrics and Child Health, University of KwaZulu-Natal, Durban, South Africa \\ ${ }^{5}$ Mpumalanga Department of Health, Nelspruit, South Africa \\ ${ }^{6}$ School of Public Health, University of the Witwatersrand, Johannesburg, South Africa
}

S Bhardwaj, ${ }^{1}$ MD, MPH; R Pattinson, ${ }^{2}$ FCOG (SA), PhD; $\mathbf{S}$ Kauchali,${ }^{3,4}$ FCPaed (SA); N Dlamini, ${ }^{3}$ MD, MMed; C Marshall, ${ }^{3}$ FCPaed(SA)

Corresponding author:S Bhardwaj (sbhardwaj@unicef.org)

Over the last few years, the South African (SA) National Department of Health has led the design, implementation and monitoring of key strategies to improve maternal, newborn, and child health outcomes. The strategies have resulted in the reduction of maternal, under-5 and infant mortality rates in SA. Here, we examine the strategies using a framework to understand the determinants and actions that influence the quality of care, coverage and resultant programme effectiveness in saving the lives of mothers and children. Three case studies from the field, namely, the Essential Steps In Managing Obstetric Emergencies, undernutrition in young children and breastfeeding are illustrated through applying the framework and demonstrating how improved quality of care and coverage achieved better health outcomes for pregnant women and children under 5 years of age. These case studies highlight different aspects of the framework, including improving healthcare workers' skills, ensuring implementation of standard protocols and strengthening management accountability within facilities and across the districts. We also highlight how these aspects collectively improved the overall programme impact. The paper concludes that the framework would be strengthened through addition of critical cross-cutting aspects related to targeting and sustainability of actions.

S Afr Med J 2018;108(3 Suppl 1):S44-S49. DOI:10.7196/SAMJ.2018.v108i3.12812

In order to have an impact at the population level, key evidence-based interventions should reach high population coverage levels, ensuring that they reach all those in need, and be delivered at high quality, meeting the standards of care. ${ }^{[1]}$ The World Health Organization (WHO) defines quality of care as the extent to which healthcare services provided to individuals and patient populations improve desired health outcomes'. In order to achieve this, healthcare must be safe, effective, timely, efficient, equitable and people-centred. ${ }^{[2]}$ Quality of care is also a key component of the right to health and the route to equity and dignity for women and children, and it is essential to deliver healthcare services that meet the quality criteria. ${ }^{[3]}$ Improving universal health coverage is also influenced by the accessibility, affordability and utilisation of the services.

Fig. 1 outlines the factors that influence programme effectiveness, including the stakeholders (individuals, the community), the enablers (healthcare managers) and the doers (healthcare providers). The framework also highlights how the various actions that influence coverage and quality of care, e.g. knowledge and the use and accessibility of the facilities, contribute to the overall impact on saving lives.

There has been progress in the reduction of maternal, under- 5 and infant mortality rates in South Africa (SA). The SA Demographic and Health Survey of 2016 (SADHS 2016) observed a decline in the under-5 mortality rate, from 59 deaths per 1000 live births (2008) to 42 deaths per 1000 live births (2016), and infant mortality from 45 deaths per 1000 live births (2008) to 35 deaths per 1000 live births (2016). ${ }^{[4]}$ The SADHS 2016 also reported that almost all live

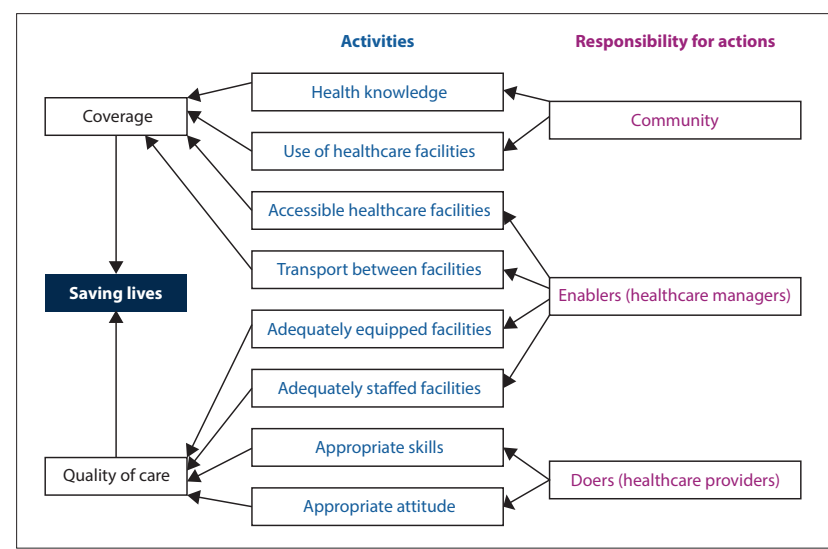

Fig. 1. Conceptual framework showing factors influencing programme effectiveness. ${ }^{[3]}$

births occurred in healthcare facilities, and that $97 \%$ of deliveries were attended by skilled healthcare workers (midwives or doctors).

Since 2009, the National Department of Health (NDoH) has led the design, implementation, and monitoring of key programme strategies and interventions for improving maternal, newborn and child health outcomes. The paper examines selected interventions in the programme effectiveness framework to understand key components for achieving coverage and quality of care in saving lives. 


\section{Methods}

We reviewed reports and strategy documents to identify interventions led by the NDoH over the last 5 years to reduce maternal and child health mortality. Specifically, we examined the interventions that focused on improving the quality of care for pregnant women and children under 5 years of age. Our aim was to select a few interventions and evaluate them against the programme effectiveness framework (Fig. 1), to understand the critical factors for success and to inform replicability and scale-up in SA.

The criteria used to select the interventions for analysis in this paper were:

1. Timeline - implemented within the timeframe of the national maternal, newborn, child and women's health and nutrition strategy $(2011$ - 2016).

2. Governance, including strategic oversight and leadership driven by the $\mathrm{NDoH}$ and not by a partner organisation.

3. Scale - implemented across an entire province or more than one province - and not in select facilities and communities only.

4. Resources - utilised existing resources in terms of human resources (no additional persons employed for implementation).

5. Quality of care activities - specific focus on improving quality of care and not only focused on coverage and achieving targets.

6. Results - interventions that showed improvement in results of maternal and child survival.

Some of the reviewed interventions included policy changes to incorporate new evidence for HIV treatment regimens, infant feeding in the context of HIV, strengthening the use of data to understand gaps in programme implementation, reducing missed opportunities for linkage and retention in care and focusing on the implementation of standard treatment guidelines for the management of malnutrition. There were also examples of the development of multisectoral implementation plans to strengthen nutrition interventions and to ensure improvement in clinical care and governance through the district clinical specialist teams (DCSTs), a group of skilled healthcare practitioners who are specifically appointed to this end.

Although all the above do contribute to overall improvement in maternal and child health outcomes, we selected interventions that met the criteria listed here for the purposes of this paper. The case studies were then examined against the programme effectiveness framework, to understand the key components and facilitators for success. They also identified additional factors that were critical to the success of the intervention beyond the components of the framework.

\section{Results}

Three interventions covering maternal and child health and survival programmes were selected and examined against the framework. For maternal health, we looked at a 12-district, two-step intervention aimed at improving obstetric skills and practices, i.e Essential Steps in Managing Obstetric Emergencies (ESMOE). For child health, we examined a province-wide, multipronged intervention that reduced the case fatality rate for severe acute malnutrition (SAM), and a nationwide, multipronged intervention to increase exclusive breastfeeding.

Achieving better maternal health outcomes through ESMOE saturation training and simulation fire drills as a quality improvement strategy

With almost all deliveries in SA occurring in health facilities, improving the quality of care provided at the facilities in a timely, consistent, safe and equitable manner should be the key focus in achieving better maternal health outcomes. The recent Saving Mothers Report ${ }^{[5]}$ identified the fact that the poor management of emergency obstetric cases was associated with maternal deaths, and recommended that it was essential to address gaps in healthcare workers' knowledge, skills and practice in order to reduce preventable deaths.

Following this recommendation, the $\mathrm{NDoH}$ developed, tested and scaled up the ESMOE training programme, in collaboration with the SA Medical Research Council. ${ }^{[6]}$ In 2012, 12 'most-in-need' districts were identified, and $>80 \%$ of the healthcare professionals that were involved in maternity care were trained in ESMOE. A 'before-and-after' assessment of the training indicated that all staff categories had improved their knowledge and skills. However, the baseline knowledge and skills were low in all categories. ${ }^{[7]}$ This indicated the need for continuing professional development for all levels of healthcare professionals.

The effect of the training on the health system was monitored by assessing the number of emergency-care signal functions a healthcare facility could perform at the start of the project and 1 year after the saturation training had been completed. A repeat baseline assessment was conducted at all the sites, using the same tools as the original baseline assessment. There were significant improvements in the number of signal functions the community healthcare centres $(p<0.01)$ and district hospitals $(p=0.05)$ could perform 1 year after the training, compared with the baseline (Table 1). However, in two areas (performing assisted deliveries and manual vacuum aspiration for spontaneous incomplete miscarriages) there was very little improvement in the community health centres (CHCs) ${ }^{[6]}$ This was due to Nursing Council regulations, which state that these activities are not within the scope of practice of professional nurses with midwifery qualifications ${ }^{[8]}$ As most of the CHCs are staffed with professional nurses with midwifery as part of their training, and not professional advanced midwives, there was very little change in these aspects.

The impact of the scale-up of the ESMOE programme was monitored by assessing the number of maternal deaths from January 2011 to the start of saturation training in each district, starting in October 2012 (the 'before' period), and from after the saturation

Table 1. Number of signal functions at baseline and repeat baseline assessment

\begin{tabular}{|c|c|c|c|c|}
\hline \multirow[b]{2}{*}{ Number of functions } & \multicolumn{2}{|c|}{ Community health centres $(n=51)$} & \multicolumn{2}{|c|}{ District hospitals $(n=62)$} \\
\hline & Baseline, \% & Repeat baseline, \% & Baseline, \% & Repeat baseline, \% \\
\hline 9 & N/A & N/A & 46.8 & 62.9 \\
\hline 8 & N/A & N/A & 80.6 & 82.3 \\
\hline 7 & 0 & 3.9 & 91.6 & 90.3 \\
\hline 6 & 5.9 & 9.8 & 96.8 & 96.3 \\
\hline 5 & 49.0 & 64.7 & 100 & 100 \\
\hline 4 & 94.1 & 100 & 100 & 100 \\
\hline 3 & 100 & 100 & 100 & 100 \\
\hline
\end{tabular}


training to the end of 2016 (the 'after' period). There was a significant reduction of $29.3 \%$ in all maternal deaths, and a $17.5 \%$ reduction in direct maternal deaths after the training compared with numbers before the training (Table 2). There was also a significant reduction in deaths due to bleeding at or after caesarean delivery. The 12 intervention districts had a $29.3 \%$ reduction in maternal deaths, compared with $5 \%$ for the other 40 districts $(p<0.0001)$.

Implementing an emergency obstetric-care training programme in which $80 \%$ of the healthcare professionals are trained was thus associated with significant improvements in the knowledge and skills of the healthcare professionals, improvement in the provision of emergency services and a reduction in maternal mortality.

\section{Sustaining and sharpening obstetric skills}

Sustaining the knowledge and skills obtained in the ESMOE programme was the next priority. An average district hospital sees approximately one woman every 2 years who dies due to obstetric haemorrhage or complications of hypertension (Table 3 ) ${ }^{[9]}$ In other words, these conditions are rare in these institutions, and even more so in CHCs. To maintain knowledge and skills to manage these women it is essential to practice by performing emergency drills.

A training programme was integrated into the ESMOE programme to teach facilities how to conduct emergency drills. This training was called Emergency Obstetric Simulation Training (EOST). The $\mathrm{NDoH}$ recommended that all maternity units (irrespective of the size of the unit) should perform at least one emergency obstetric drill per month. These drills were recognised for continuing professional development $(\mathrm{CPD})$ points if there was a clear record of them being performed. A record of having performed the drill had to be submitted to a central office.

Facilities that performed regular drills, as evidenced by their submission for CPD points, were analysed, and a significant correlation was found between the reduction in maternal mortality and the number of drills. Those districts where drills were conducted and recorded regularly had an $18.4 \%$ reduction in mortality, whereas those who did not only had a $5.2 \%$ reduction in mortality (Table 4).$^{[10]}$ The training in emergency drills continues to be expanded throughout the country in all maternity units. The aim of the training is for every maternity unit to have staff trained in emergency care, and to conduct regular emergency drills.

Analysing this intervention against the programme effectiveness framework shows that the focus was on improving the quality of care and ensuring appropriate skills in the healthcare providers, i.e. the doers, in the framework. However, a critical aspect of success was the focus on sustaining the skills, by institutionalising their implementation and linking these to incentives, i.e. the CPD points system. The incorporation of sustainability as an important crosscutting component in the programme-effectiveness framework is further highlighted in the discussion section of the paper.

\section{Strategies to improve under- 5 survival of by improving nutritional outcomes}

There are no direct population estimates of the contribution of undernutrition or acute malnutrition to under- 5 mortality in SA. However, clinical mortality audit data routinely collected in public hospitals indicates that at least $50 \%$ of under- 5 deaths in hospitals

Table 2. Comparison of all maternal deaths and direct maternal deaths before and after ESMOE and EOST saturation training

\begin{tabular}{|c|c|c|c|c|c|}
\hline & Live births & All MD* & iMMR (all) & 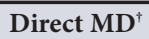 & Direct iMMR \\
\hline Before & 645477 & 1332 & 206.4 & 628 & 97.30 \\
\hline After & 602856 & 880 & 146.0 & 475 & 78.79 \\
\hline
\end{tabular}

Table 3. Distribution of births per facility per year

\begin{tabular}{|c|c|c|c|c|c|c|}
\hline & \multirow[b]{2}{*}{ Facilities, $n$} & \multicolumn{3}{|c|}{$\begin{array}{l}\text { Number of pregnant women who died after } \\
\text { giving birth, per level of care }\end{array}$} & \multirow[b]{2}{*}{ Births/year } & \multirow[b]{2}{*}{ Average births/facility } \\
\hline & & NPRI & $\mathrm{OH}$ & HT & & \\
\hline $\mathrm{CHC}^{*}$ & ND & 125 & 95 & 112 & 172984 & ND \\
\hline $\mathrm{DH}$ & 256 & 166 & 116 & 111 & 392861 & 1535 \\
\hline $\mathrm{RH}$ & 48 & 139 & 81 & 87 & 239269 & 4985 \\
\hline PT & 16 & 70 & 42 & 61 & 74066 & 4629 \\
\hline NC & 8 & 23 & 14 & 20 & 58497 & 7312 \\
\hline
\end{tabular}

Table 4. Effect of EOST exercises

\begin{tabular}{|c|c|c|c|c|c|c|}
\hline $\begin{array}{l}\text { Submitted data } \\
\text { for CPD points }\end{array}$ & Districts, $n^{*}$ & $\begin{array}{l}\text { Average iMMR } \\
2011 \text { - } 2013 \\
\text { (before EOST) }\end{array}$ & $\begin{array}{l}\text { Average number } \\
\text { of drills per } \\
\text { district }\end{array}$ & $\begin{array}{l}\text { Average iMMR } \\
2014-2015 \\
\text { (after EOST) }\end{array}$ & Average difference & $\begin{array}{l}\text { Percentage } \\
\text { decrease }\end{array}$ \\
\hline No & 25 & 134.8 & 0 & 127.8 & 7.0 & 5.2 \\
\hline Yes & 20 & 174.3 & 145 & 142.3 & 32.1 & $18.4^{+}$ \\
\hline Total & $45^{*}$ & 152.4 & 64 & 134.3 & 18.1 & 11.5 \\
\hline
\end{tabular}


were associated with underweight status, and just over 30\% were attributable to severe acute malnutrition (SAM). ${ }^{[1]]}$ The SADHS 2016 showed that there has been a remarkable improvement in exclusive breastfeeding, from 7\% in 1998 to $32 \%$ in 2016. ${ }^{[4]}$ However, this still means that two out of three children are not exclusively breastfed and miss the benefits of this practice. The sections below describe the two selected interventions that led to improvement in child survival outcomes, and examine these interventions against the programmeeffectiveness framework.

Improving inpatient quality of care of children with severe acute malnutrition (SAM)

All children aged under 5 should be assessed, with growth monitoring and plotting of the growth curve at every visit at the primary-care level, and referred, where necessary, to higher levels of care. Improved, timely and accurate case detection of all forms of acute malnutrition, accompanied by better data reporting, will result in more SAM cases that access early nutritional treatment, care and support. Early access to treatment would deliver better outcomes in these cases.

To reduce overall inpatient under-5 mortality, the NDoH prioritised the development and implementation of quality improvement plans to decrease the case fatality rate for SAM. The plans included the formation of a multidisciplinary management team led by senior hospital management; improving active case detection in casualties and inpatient care units; strengthening healthcare workers' skills to treat and manage sick SAM cases by improving adherence to standard treatment guidelines; and improving data quality through better recording, reporting and responding to avoidable factors contributing to SAM deaths.

The inpatient SAM case fatality rates (iCFRs) in SA provide a good composite proxy measure of the quality of care provided to sick SAM inpatients. Fig. 2 shows the trends in the iCFRs from 2009 to 2017, and there is a clear systematic reduction in the SAM case fatality rate from 2009 to 2016, with a significant reduction observed in the last 3 years of intensive implementation of the quality improvement plan; this effort targeted facilities with the largest number of SAM deaths.

To illustrate the impact of the national intervention, we describe a case study from the province of Mpumalanga. The case fatality rate in Mpumalanga province between 2011/12 and 2014/15 ranged between $15 \%$ and $25 \%$.

Fig. 3 shows the trends in the SAM iCFRs in under- 5 children from 2011/12 - 2016/17 in Mpumalanga. Following an initial reduction in the provincial and district SAM iCFRs from 2011 to 2013, an increase in the rates occurred during the 2014/15 financial year, to $19.1 \%$, with 233 children dying from SAM-related mortality. In order to address this high mortality rate, the province developed a turnaround

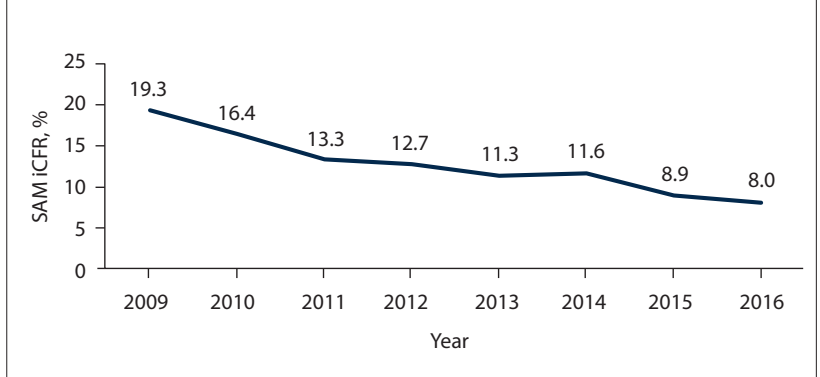

Fig. 2. Trends in the SAM inpatient CFRs between 2009 and 2017. (SAM = severe acute malnutrition; $i C F R=$ inpatient case fatality rate.)

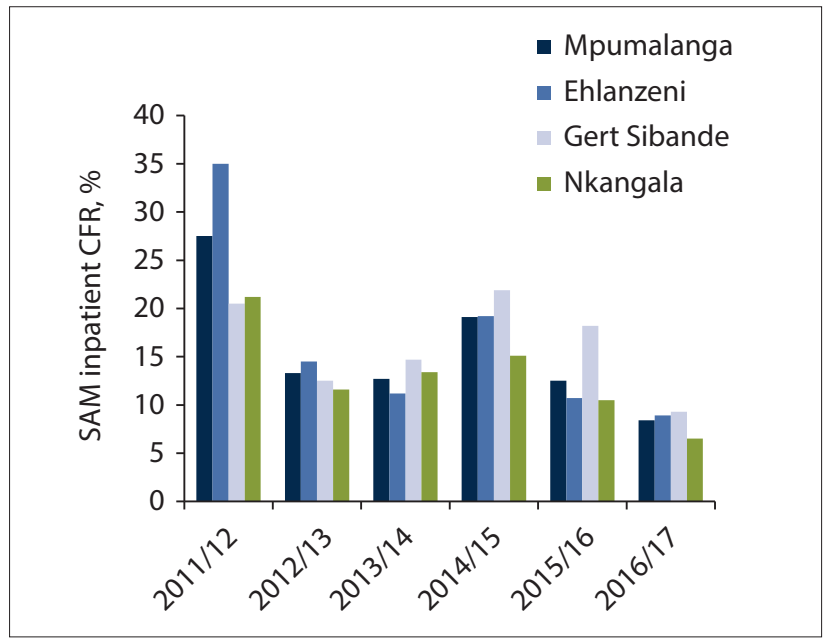

Fig. 3. Provincial- and district-level SAM inpatient CFR for under-5 children in Mpumalanga: 2011/12 - 2016/17. (SAM = severe acute malnutrition; $C F R=$ case fatality rate. Data obtained from the District Health Information System.)

strategy with support the $\mathrm{NDoH}$, implementing partners and the University of Pretoria.

Fig. 4 highlights the different activities implemented over a period of 2 years (2015 - 2017). These activities were aimed at improving early case detection, referral and appropriate treatment and management of SAM cases, as needed. An analysis of the interventions against the programme-effectiveness framework highlighted the following aspects:

1. A focus on coverage, with activities in the framework including: a. Health promotion to improve health knowledge and use of facilities by communities.

b. Improving access to healthcare facilities and transport these important aspects were facilitated by the enablers (healthcare managers).

2. A focus on quality of care, with activities in the framework including:

a. Adequately equipped facilities and adequately staffed facilities. This was done by enablers (healthcare managers).

b. Appropriate skills and appropriate attitudes - ensured by doers (healthcare providers).

All the above was made possible through management oversight and using evidence-based planning involving a multidisciplinary team. The interventions collectively contributed to an overall provincial reduction in SAM mortality to 146 deaths during 2015/16 (a decrease of $12.5 \%$ in the iCFR), which was subsequently reduced to 83 deaths (a further decrease of $7.8 \%$ in the iCFR) during 2016/17. The most notable reduction in the iCFR (from 21.9\% during 2014/15 to $9.3 \%$ in 2016/17) and the number of inpatient SAM deaths (from 82 deaths during 2014/15 to 19 deaths during 2016/17) occurred in the Gert Sibande District.

Creating an enabling policy and implementation environment to improve the coverage of breastfeeding

Improving infant and young-child feeding practices requires concerted efforts towards improving breastfeeding rates in SA. Breastfeeding remains the undisputed preferred feeding option for optimal growth and development of children. The breastfeeding series published in the Lancet in 2016 reinvigorated interest in breastfeeding and, in addition to the well-known advantages of 


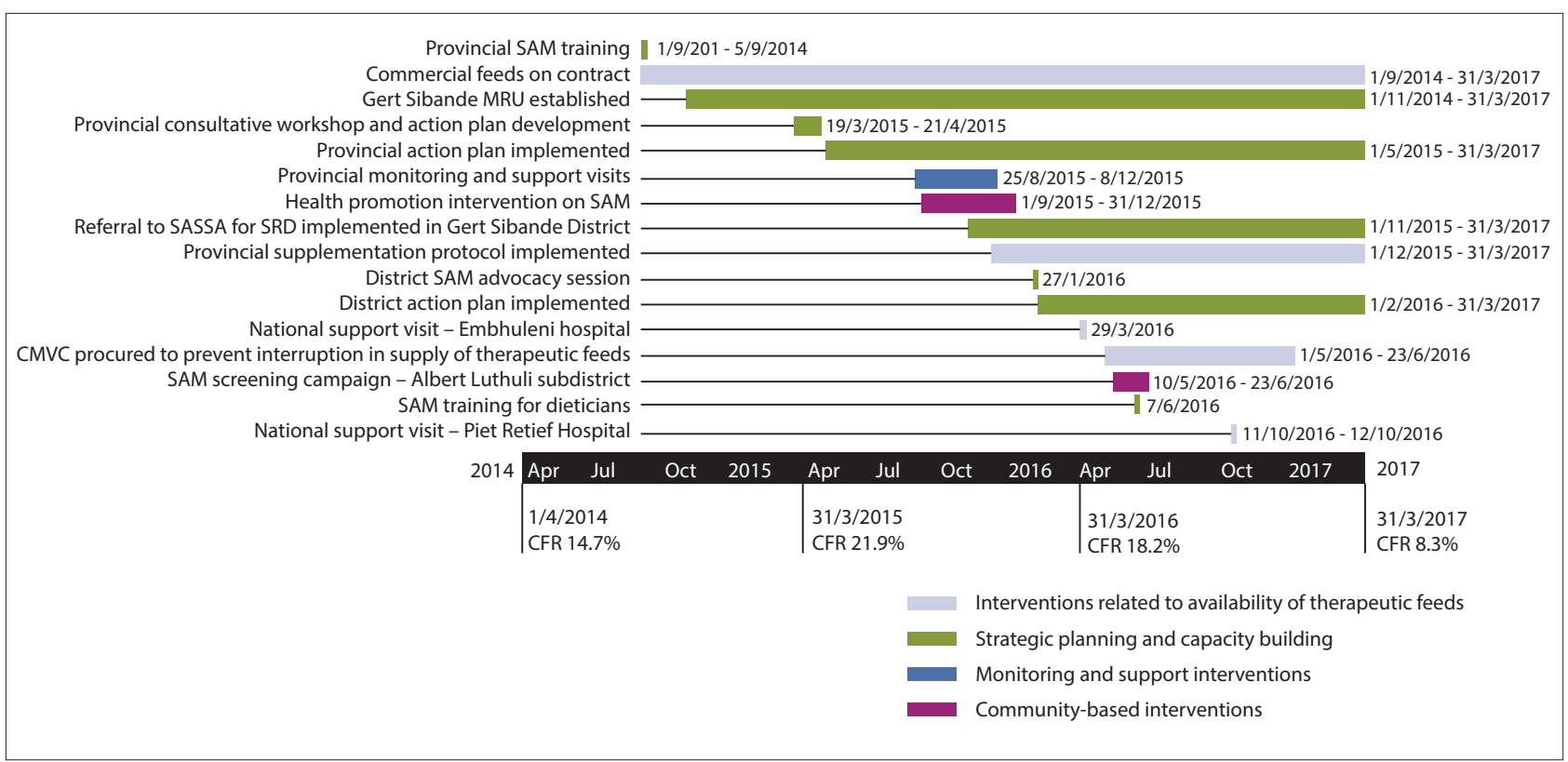

Fig. 4. Timeline of interventions aimed at improving the integrated management of under-5 children with severe acute malnutrition in Mpumalanga between 2014 and 2017. (SAM = severe acute malnutrition; $M R U=$ monitoring and response unit; SASSA = South African Social Security Agengy; SRD = social relief of distress; $C M V C=$ combined mineral vitamin complex; $C F R=$ case fatality rate.)

breastfeeding, also highlighted the long-term breastfeeding outcomes of improved intelligence and protection against the development of obesity and diabetes. ${ }^{[12]}$ The World Bank has identified breastfeeding as the most lucrative return on investment, and noted the additional benefits to the mother, including fertility protection and reduced risk of the development of breast and ovarian cancer. ${ }^{[13]}$

Historically, breastfeeding became less common in high-income countries during the 20th century, and this had a ripple effect in lower- and middle-income countries. Breastmilk substitutes (infant formula) were viewed as modern and prestigious. SA did not escape this trend, and it was exacerbated by the provision of free infant formula as part of the prevention of mother-to-child transmission programme. The detrimental effects of this practice were well documented.

In a laudable attempt to reposition breastfeeding as a child survival strategy, and to do away with formula feeding, SA adopted the Tshwane Declaration on Breastfeeding in August 2011, with highlevel political support from the Minister of Health. ${ }^{[14]}$ The declaration reaffirmed the fact that breastfeeding rates in SA were extremely low. In addition to calling for the prohibition of free formula, it called for the establishment of human-milk banks, the implementation of the MBFI (Mother Baby Friendly Hospital Initiative) and Kangaroo Mother Care. Currently, there are 37 breastmilk banks, and 407 out of $545(75 \%)$ public health facilities with maternity beds are accredited as part of the MBFI. In December 2016, SA cohosted the World Breastfeeding Conference. The deputy Minister of Health launched the country's first 3-year Breastfeeding Campaign during the opening ceremony of the conference. ${ }^{[15]}$

The interventions are beginning to bear fruit, as evidenced by the cumulative exclusive breastfeeding rate, which has increased from $7 \%$ in 1998 to $32 \%$ in $2016 .{ }^{[4]}$ SA now wants to accelerate this rate, and has set an ambitious target of increasing its 14-week exclusive breastfeeding rates to $70 \%$ in 2019 .

Reviewing the national scale-up of the promotion of exclusive breastfeeding over the last few years, with interventions by the community, enablers and doers based on the programme- effectiveness framework, showcases the multiplicity of actions to influence coverage and quality of care. The milk banks and the MBFI scale-up ensured that there were adequately equipped facilities and staff with appropriate skills and attitude, while the campaigns and community engagement focused on actions at the community level and improving health knowledge and the use of healthcare facilities.

\section{Discussion}

The interventions highlighted in this article demonstrate how selected interventions have contributed to the reduction in maternal and child deaths through improvement in the quality of care and coverage.

We found that access to services (ensured by the enablers) and their use (by individuals) is an important component of overall quality of care. The sustained use of the services (based on improved actions and attitudes of healthcare providers) was an important component of coverage and quality. We do know that provision of equipment and adequate staffing are critical aspects of quality of care. These were not covered in the case studies, except for the issue of staffing, as highlighted in the maternal-health example. The lack of adequate staffing is a barrier to providing emergency obstetric care, and it is important to find a solution to ensure a sustained improvement in effective coverage. ${ }^{[16]}$

While key actions focused on coverage and quality of care are covered in all three interventions, we found limitations in the programme-effectiveness framework.

In relation to the 'doers', the article does identify challenges with appropriately skilled staff (noting the low baseline knowledge and skills levels with respect to obstetric emergencies) and the reliable implementation of protocols by staff (in relation to inadequate treatment of severely malnourished children). Basic training needs to ensure that graduates are competent. In addition, there are large numbers of existing staff, foreign-trained and sessional doctors in the country, which means that initiatives such as ESMOE, aided perhaps by new technologies such as e-learning, will be needed at a larger scale and with a guaranteed level of quality for sustainability in generating results. 
In relation to the community and the individual, health knowledge appears to be variable, and therefore adequate childcare practices, especially the sustaining of breastfeeding, remain challenging. The establishment of a multisectoral forum to address the multifaceted problems of under- and over-nutrition is important. However, the low (though improving) breastfeeding rates and relatively high levels of stunting all point to the need for more effective actions in this area.

The observed successes have been a consequence of important initiatives in policy and programme design, including supporting rollout and implementation of the initiatives. However, an important point to note is the high level of political commitment to and technical oversight of these initiatives in SA, which was demonstrated by the setting up of ministerial advisory committees for each target group, i.e. mothers, newborns and children. These committees are guided by passionate and knowledgeable expert-advocates, who are able to analyse information and recommend the latest evidencebased action. They benefit from the direct support and dedication of the Minister of Health

The framework does not, however, account for key cross-cutting issues, such as political commitment, allocation of resources and overall management oversight, that are critical to ensuring sustainability for both coverage and quality of care.

Furthermore, the importance of focusing the actions by using relevant data and targeting the initiatives to areas that are most in need of positive impacts on survival is seen in the examples described in this article. The emphasis on understanding the evidence and ensuring that data collected are analysed, interpreted and used for action has been a major thrust across the programmes. In addition, understanding the context and building on recommendations in the midterm review of the maternal, newborn child and women's health programmes in $2014^{[17]}$ led to the focus on clinical governance issues and improving local motivation, management skills and efforts to maximise partnerships with academia, professional bodies and partner organisations. Efforts were made to ensure that local-level data resulted in actions tailored to the local context, as seen in the emphasis and prioritisation of specific activities in the phased scale-up of the ESMOE training programme and the case study from Mpumalanga.

In SA, clinical experts were placed at the district level to analyse the local situation and develop targeted solutions. Initiatives such as ESMOE and EOST, and improved SAM case management, were possible because of the maximal utilisation of these skilled cadres.

The use of the framework would benefit by adding a fourth dimension of cross-cutting components. These include evidencebased targeting and the use of data for action, management oversight towards institutionalising and sustaining actions, continuous feedback loops through monitoring and mentoring actions, forging and sustaining partnerships, leveraging on existing resource and partner networks, and resource allocations. All of these were highlighted in the examples described in the article and contributed to the success of the interventions and overall impact on improving maternal- and child-health outcomes.

\section{Conclusion}

Significant improvements in programme effectiveness, with better coverage and quality of care, were achieved. This has resulted in a reduction in the number of deaths and an improvement in early identification of weaknesses and consequent change of practices. However, more needs to be done towards sustaining these efforts and institutionalising these practices.
The global agenda of the Sustainable Development Goals (SDGs) for 2030 on maternal and child health carries the slogan 'Survive, thrive and transform. ${ }^{\text {'[18] }}$ In SA, the new Integrated Plan for Reducing Mortality in Mothers, Newborns and Under-fives has a strong focus on reducing mortality in healthcare facilities that underpins the goals of the 'thrive' component of the SDGs. Reflecting on the enhanced programme effectiveness framework highlights the importance of interventions that address the social determinants of healthcare, especially among the most vulnerable, and sustained improvements in client experiences of healthcare service delivery, including focusing on the empowerment of mothers, caregivers and staff at facilities.

Acknowledgements. The authors wish to acknowledge SAMRC for the support of the Maternal and Infant Health Care Strategies Unit and the provincial Department of Health for supporting the quality improvement plans.

Author contributions. RB, PB, SB, SK conceptualised the paper. RB wrote the initial draft and different sections were written by SK, MV, ND, CM. SB collated and finalised the paper, $\mathrm{PB}$ and SK supported review, editing and submission of the first draft. $\mathrm{PB}, \mathrm{SB}, \mathrm{SK}, \mathrm{CM}, \mathrm{RB}, \mathrm{ND}$ provided significant inputs to the paper. SB finalised and edited the paper, SK and $\mathrm{PB}$ reviewed and edited the manuscript.

Funding. None.

Conflicts of interest. None.

1. Tanahashi, T. Health service coverage and its evaluation. Bull World Health Organ 1978;56(2):295-303. 2. World Health Organization. Quality of Care. Geneva: WHO, 2017.

3. Save the Children, United Nations Children's Fund, National Department of Health, South African Medical Research Council/University of Pretoria Maternal and Infant Health Care Strategies Unit. Every Death Counts. Saving the lives of mothers, babies and children in South Africa. Cape Town: Every Death Counts. Saving the lives of mothers, babies and children in South Afric
SAMRC, 2008. http://www.mrc.ac.za/bod/edcrptfinal.pdf (accessed 1 February 2018).

4. Statistics South Africa. South Africa Demographic and Health Survey 2016: Key Indicators Report, 2016. Pretoria: StastSA, 2017

5. National Committee for Confidential Enquiries into Maternal Deaths. Saving Mothers 2005 2007: Fourth Report on Confidential Enquiries into Maternal Deaths in South Africa. - 2007: Fourth Report on Confidential Enquiries into Maternal Deaths in South Africa.
Pretoria: NCCEMD, 2008. http://wwwhealth.gov.za/index.php/2014-08-15-12-55-04/category/101$2011 \mathrm{rp}$ ? download=190: saving-mothers-2005-2007-fourth-report-on-confidential-enquiries-intomaternal-deaths-in-south-africa-full-report (accessed 1 February 2018).

6. Frank K, Lombaard H, Pattinson R. Does completion of the Essential Steps in Managing Obstetric Emergencies (ESMOE) training package result in improved knowledge and skills in managing obstetric emergencies? S Afr J Obstet Gyn 2009;15(3):94-99.

7. South African Medical Research Council. Essential Steps in Managing Obstetric Emergencies (ESMOE) - Emergency Obstetric Simulation Training (EOST) Scale-up Programme: Report to National Department of Health. Pretoria: SAMRC, 2016.

8. National Department of Health. Nursing Act, 2005 (Act No. 33 of 2005). Regulations relating to the approval of and the minimum requirements for the education and training of a learner leading to registration in the category midwife. Government Notice No. R786. 2014.

9. National Committee for Confidential Enquiries into Maternal Deaths. Saving Mothers 2011 - 2013: Sixth Report on Confidential Enquiries into Maternal Deaths in South Africa. Pretoria: NCCEMD, 2014. http://www.kznhealth.gov.za/mcwh/Maternal/Saving-Mothers-2011-2013-short-report.pdf 2014. http://www.kznh

(accessed 1 February 2018)

10. Pattinson R. Correlation between numbers of EOST drills reported for CPD points and change in iMMR per district. Priorities in Perinatal Care Conference, Mpekweni Holiday Resort, Eastern Cape, South Africa, 7 - 10 March 2017. https://www.perinatalpriorities.co.za/proceedings-database/ Presentation (accessed 1 February 2018).

11. Stephen CR, Bamford LJ. Saving Children 2010 - 2011: A Seventh Survey of Child Health Care in South Africa. Pretoria: Tshepesa Press, Medical Research Council and Centers for Disease Control and Prevention, 2013:1-142.

12. Rollins NC, Bhandari N, Hajeebhoy N, et al. Why invest, and what it will take to improve breastfeeding practices? Lancet 2016;387(10017):491-504. https://doi.org/10.1016/S0140-6736(15)01044-2

13. Shekar M, Kakietek J, Eberwein JD, Walters D. An Investment Framework for Nutrition: Reaching the Global Targets for Stunting, Anemia, Breastfeeding, and Wasting. Washington: The World Bank, 2017.

4. National Department of Health. The Tshwane Declaration of Support for Breastfeeding in South Africa. S Afr J Clin Nutr 2011;24(4):214.
Aational Department of Health. The Thu

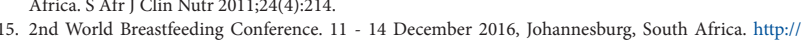
worldbreastfeedingconference.org/agenda.html (accessed 1 February 2018).

16. Donabedian A. The quality of care. How can it be assessed? JAMA 1998;260(12):1743-1748. https:// doi.org/10.1001/jama.1988.03410120089033

17. National Department of Health. Mid-term Review of Strategic Plan for Maternal, Newborn, Child and Women's Health and Nutrition in South Africa 2012 - 16. Pretoria: NDoH, 2016. http:// childhealthpriorities.co.za/conference-presentations/2014/Review $\% 20$ of $\% 20$ the $\% 20$ National $\% 20$ MNCWH\%20and\%20N\%20Strategy\%202012\%202016\%20S\%20Bardwaj\%20H\%20Saloojee.pdf (accessed 1 February 2018)

18. World Health Organization. Global Strategy for Women's, Children's and Adolescents' Health, 2016-2030. Geneva: WHO, 2017.

Accepted 10 October 2017 
